\title{
HYPERMETRIC SPACES AND THE HAMMING CONE
}

\section{DAVID AVIS}

1. Definitions and preliminary results. We denote by $d=\left(d_{12}\right.$, $\left.\ldots, d_{1 n}, d_{23}, \ldots, d_{n-1, n}\right)$ a vector of $\left(\begin{array}{l}n \\ 2\end{array}\right)$ distances between $n$ points. Such a vector $d$ is called a metric if it satisfies the triangle inequalities

(1) $d_{i j}+d_{j k} \geqq d_{i k} \quad 1 \leqq i, j, k \leqq n$.

The set of all metrics on $n$ points forms a convex polyhedral cone, the extremal properties of which are discussed in [4]. We will be concerned with a sub-cone that is spanned by metrics of the form

$$
d_{i j}(t)=\left\{\begin{array}{cc}
t & i \perp j \in V \\
0 & \text { otherwise },
\end{array} \quad 1 \leqq i<j \leqq n\right.
$$

where $t \geqq 0, V$ is a proper subset of $\{1,2, \ldots, n\}$ and the symbol $\perp$ is used for "exclusive or": $i \perp j \in V$ means $i \in V, j \notin V$ or $i \notin V, j \in V$. The metrics (2) are extreme rays of the metric cone and are called Hamming rays. The convex hull of these rays is called the Hamming cone $H_{n}$ and we call $d$ Hamming, if $d \in H_{n}$. Such metrics are also called $L^{1}$-embeddable (e.g., [2]) or addressable (e.g., [5]).

Let $\Omega$ be a finite set and let $\left\{A_{i} \mid 1 \leqq i \leqq n\right\}$ be a collection of $n$ subsets of $\Omega$ that will be called addresses. Then it can be shown that a metric $d$ is Hamming if and only if for some finite set $\Omega$, there exist addresses $A_{i}$ and non-negative weights $w_{j}(j \in \Omega)$ so that

$$
d_{i j}=\sum_{k \in A_{i} \Delta A_{j}} w_{k},
$$

where $\Delta$ denotes symmetric difference. In the case where the weights are binary valued, the metrics are just the usual Hamming metrics that appear in coding theory.

Let $F_{n}$ denote the $\left(2^{n-1}-1\right)$-tuple of all proper subsets of $\{1,2, \ldots, n\}$ that contain the element 1 . Then from the definition, $d$ is Hamming if and only if the following primal problem has a solution:

$$
\begin{array}{cc}
P . \sum_{\substack{S \in F_{n} \\
i \perp j \in S}} \lambda_{S}=d_{i j} & 1 \leqq i<j \leqq n \\
\lambda_{S} \geqq 0 & S \in F_{n} .
\end{array}
$$

Received June 27, 1979 and in revised form February 16, 1981. This research was supported by the Natural Sciences and Engineering Research Council under grant A-3013 and by the National Science Foundation under grant MCS76-81259. 
This formulation has two consequences. First, if $P$ has a solution it has a solution that uses at most $\left(\begin{array}{l}n \\ 2\end{array}\right)$ non-zero variables. Thus we may assume that $|\Omega| \leqq\left(\begin{array}{l}n \\ 2\end{array}\right)$ and that the address lengths are similarly bounded. Second, an application of Farkas' Lemma (see [13]) states that $P$ is feasible if and only if the following system is infeasible:

$$
\begin{gathered}
\text { D. } \sum_{i \perp j \in S} y(i, j) \leqq 0 \quad S \in F_{n} \\
y \cdot d>0 .
\end{gathered}
$$

A vector satisfying (4) is called dual feasible. Let $B$ denote the set of all facets (maximum dimensional faces) of $H_{n}$, so that

$$
H_{n}=\{d \mid b . d \leqq 0, b \in B\} .
$$

If $z_{S}$ is the Hamming ray that corresponds to some $S \in F_{n}$, and $b \in B$, then $b z_{S} \leqq 0$ reduces to

$$
\sum_{i \perp j \in S} b(i, j) \leqq 0 .
$$

Therefore dual feasible vectors are facets if $\left(\begin{array}{l}n \\ 2\end{array}\right)-1$ linearly independent constraints in (4) are satisfied as equations.

We now consider vectors $y$ that have the form $y(i, j)=c_{i} c_{j}$ for suitably chosen real numbers $c_{1}, c_{2}, \ldots, c_{n}$. In this case, if

$$
\sum_{i=1}^{n} c_{i}=t
$$

then row $(i, j)$ of $(4)$ becomes

$$
\begin{aligned}
\sum_{i \perp j \in S} c_{i} c_{j} & =\sum_{i \in S} c_{i}\left(\sum_{j \notin S} c_{j}\right)+\sum_{i \notin S}\left(\sum_{j \in S} c_{j}\right) \\
& =2\left(\sum_{i \in S} c_{i}\right)\left(t-\sum_{i \in S} c_{i}\right) \leqq 0 .
\end{aligned}
$$

This proves the following theorem.

Theorem 1.1. If there exist real numbers $c_{1}, c_{2}, \ldots, c_{n}$ with sum $t \geqq 0$ satisfy

(i) $\sum_{i \in S} c_{i} \leqq 0$ or $\sum_{i \in S} c_{i} \geqq t$ for all $S \in F_{n}$, and

(ii) $\sum_{1 \leqq i<j \leqq n} c_{i} c_{j} d_{i j}>0$,

then $d \notin H_{n}$. 
Kelly [11] calls a metric space, $d$, hypermetric if for all integers $c_{1}, \ldots, c_{n}$ which sum to one,

(5) $\sum_{1 \leqq i<j \leqq n} c_{i} c_{j} d_{i j} \leqq 0$.

The notion of hypermetricity, but not the term, seems to have been first introduced by Deza [8]. Theorem 1.1 has the following corollary, which has been proved independently by Deza [7], [8] and Kelly [11].

COROLlaRY 1.2. If $d \in H_{n}$ then $d$ is hypermetric.

Observe that the triangle inequality is obtained from (5) by setting $c_{i}=c_{j}=1$ and $c_{k}=-1$ for $i, j, k \in\{1,2, \ldots, n\}$. This is the first of a series of inequalities, the next being the pentagon inequality, see [7] and [11], where three indices are set to +1 and two indices are set to -1 . The reader interested in a full treatment of hypermetric spaces is referred to [12]. In the next section we show that the converse of Corollary 1.2 is false in general and give some specific instances when it is true. We also exhibit a facet of $H_{n}$ that does not have the form of Theorem 1.1.

2. Main results. This section deals with the converse of Corollary 1.2. First, Deza [7] has shown that every 5 point hypermetric is Hamming. Second, as we now demonstrate, a theorem of Djoković [9] can be used to prove that all hypermetric bi-partite graphs are Hamming under the normal shortest distance metric for graphs. Indeed, following Djoković, a subset $V_{0}$ of vertices of a bi-partite graph $G$ is closed if for every $a$ and $b$ contained in $V_{0}$ and any vertex $w$ satisfying

$$
d_{G}(a, w)+d_{G}(w, b)=d_{G}(a, b)
$$

we have $w \in V_{0}$. Here, $d_{G}$ is the shortest distance metric induced by the graph $G$. For every edge $a b$, let $G(a, b)$ denote the set of points closer to $a$ than $b$. Note that the fact that $G$ is bipartite implies that if $w \notin G(a, b)$, then $w \in G(b, a)$, for any vertex $w$.

Theorem 2.1. (Djoković [9], see also [5]) For a connected bi-partite graph $G, d_{G}$ is Hamming if and only if $G(a, b)$ is closed for adjacent vertices $a$ and $b$.

We now show that this theorem has the following corollary.

Corollary 2.2. For a connected bipartite graph $G=(V, E), d_{G}$ is Hamming if and only if the pentagon inequality is satisfied.

Proof. $(\Rightarrow)$ Assume $d_{G} \notin H_{n}$. By Theorem 2.1 there exist adjacent vertices $a, b \in V$ with $G(a, b)$ not closed. Therefore there exists $u$, $v \in G(a, b)$ and $w \in G(b, a)$ with

$$
d_{G}(u, w)+d_{G}(w, v)=d_{G}(u, v) .
$$


Now $u \neq a$, for otherwise

$$
\begin{aligned}
d_{G}(a, v)=d_{G}(a, w)+d_{G}(w, v)=1+d_{G}(b, w)+ & d_{G}(w, v) \\
& \geqq 1+d_{G}(b, v)
\end{aligned}
$$

and so $v \notin G(a, b)$, a contradiction. Similarly $v \neq a$. Also $w \neq b$, for otherwise

$$
d_{G}(u, v)=d_{G}(u, b)+d_{G}(b, v)>d_{G}(u, a)+d_{G}(a, v),
$$

violating the triangle inequality. We must therefore have the situation in Figure 2.1. Let

$$
c_{i}=\left\{\begin{array}{rl}
1 & i=u, b, v \\
-1 & i=a, w \\
0 & \text { otherwise. }
\end{array}\right.
$$

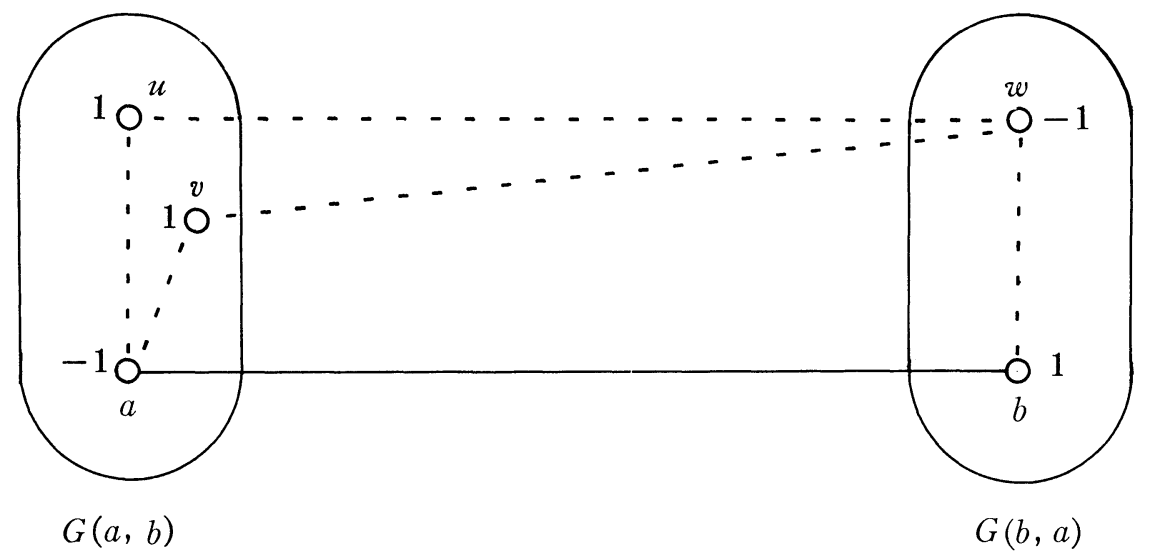

FIGURE 2.1

Note that $d_{G}(u, b)=1+d_{G}(u, a)$ since $u \in G(a, b)$. Using similar simplifications we have

$$
\begin{aligned}
\sum c_{i} c_{j} d_{G}(i, j)= & {\left[3+d_{G}(u, a)+d_{G}(v, a)+d_{G}(b, w)\right] } \\
& -\left[1-d_{G}(u, a)+d_{G}(v, a)+d_{G}(b, w)\right] \\
= & 2>0 .
\end{aligned}
$$

Therefore $d_{G}$ violates the pentagon inequality and is not hypermetric.

Complete results for graphs in general are known only for $|G| \leqq 6$. An examination of graphs with 6 or fewer vertices (listed in [10]) produced the five minimal nonhypermetric graphs shown in Figure 2.2. The integers attached to the vertices of the graphs in Figure 2.2 correspond to the integers $c_{1}, c_{2}, \ldots, c_{n}$ that form the coefficients of the hypermetric inequality (5) that is violated. All nonHamming graphs with 6 or fewer 


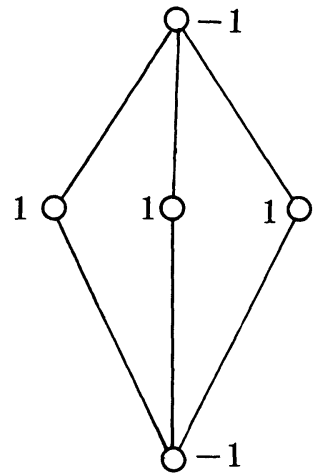

(a)

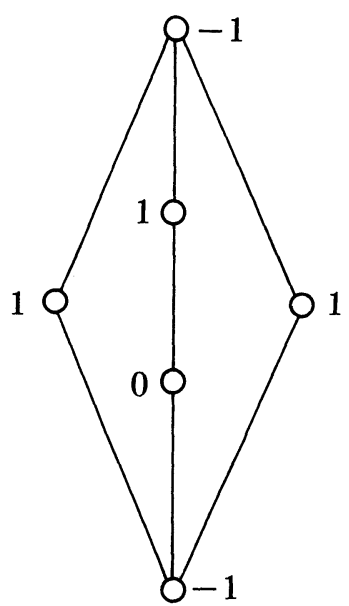

(d)

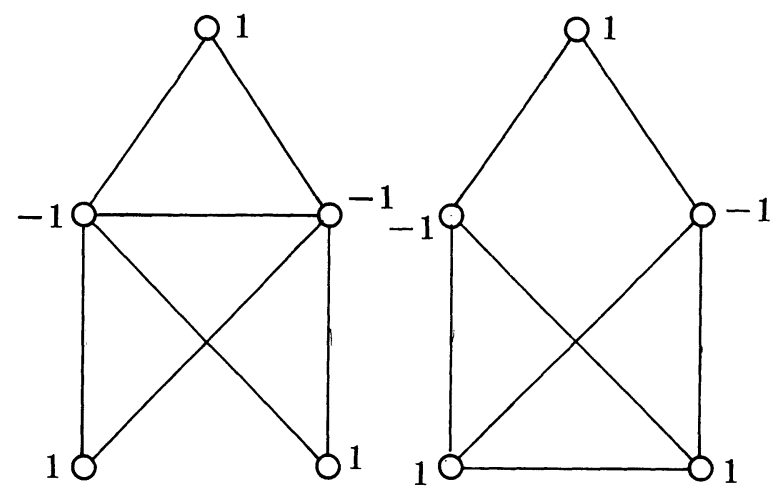

(b) (c)

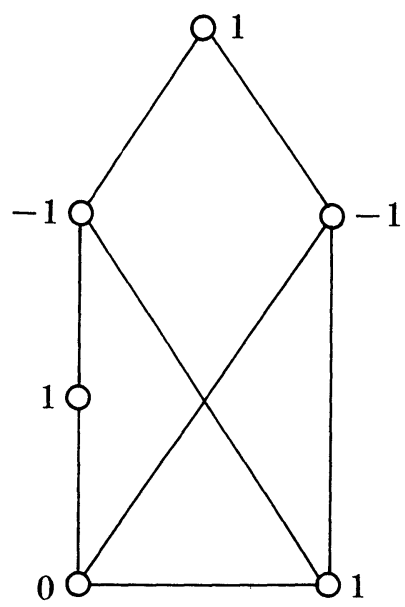

(e)

FIGURE 2.2. All minimal nonhypermetric graphs on 6 or fewer vertices

vertices contain an isometric nonhypermetric subgraph. Thus the converse of Corollary 1.2 is true for graphs $G$ with $|G| \leqq 6$. For $|G| \leqq 5$, this also follows from results in [7].

We now show that the converse to Corollary 1.2 is false for $|G| \geqq 7$, by exhibiting a nonHamming hypermetric graph. Consider the graph $G$ formed from $K_{7}$ by deleting edges $v_{1} v_{2}$ and $v_{1} v_{3}$.

Theorem 2.3. $G$ is a nonHamming hypermetric graph.

Proof. We begin by proving that $d_{G}$ is hypermetric. Assume conversely that there exists integers $c_{1}, c_{2}, \ldots, c_{7}$ such that

$$
\sum_{i=1}^{7} c_{i}=1 \text { and } \sum_{1 \leqq i<j \leqq 7} c_{i} c_{j} d_{G}(i, j) \geqq 1 .
$$


Using the identity

$$
2 \sum_{1 \leqq i<j \leqq 7} c_{i} c_{j}=\left(\sum_{i=1}^{7} c_{i}\right)^{2}-\sum_{i=1}^{7} c_{i}{ }^{2}
$$

and the structure of $G$ we have

$$
2 \sum_{1 \leqq i<j \leqq 7} c_{i} c_{j} d_{G}(i, j)=2 c_{i}\left(c_{2}+c_{3}\right)+1-\sum_{i=1}^{7} c_{i}{ }^{2} .
$$

Therefore $c_{1}, \ldots, c_{7}$ satisfy

(6) $2 c_{1}\left(c_{2}+c_{3}\right) \geqq \sum_{i=1}^{7} c_{i}{ }^{2}$.

Consider the function $f$ defined over integer vectors $c=\left(c_{1}, \ldots, c_{7}\right)$ by

$$
f(c)=2 c_{1}\left(c_{2}+c_{3}\right)-\sum_{i=1}^{7} c_{i}{ }^{2} .
$$

Set $k=c_{1}+c_{2}+c_{3}$ and define $g$ by

$$
g\left(c_{1}, c_{2}, c_{3}\right)=2 c_{1}\left(c_{2}+c_{3}\right)-c_{1}{ }^{2}-c_{2}{ }^{2}-c_{3}{ }^{2}-(1-k)^{2} / 4 .
$$

Then $g$ bounds $f$ from above for fixed $c_{1}, c_{2}$ and $c_{3}$ since it sets $c_{4}=c_{5}=$ $c_{6}=c_{7}$ (their optimal values). We are interested in the values of $k$ that allow $g$ to be nonnegative. To this end we seek to solve the quadratic program

$$
\max g\left(c_{1}, c_{2}, c_{3}\right)
$$

subject to

$$
c_{1}+c_{2}+c_{3}=k .
$$

Inspection shows that, for fixed $k$, the maximum occurs for $c_{2}=c_{3}$. Making the indicated substitutions,

$$
g\left(c_{1}, c_{2}, c_{3}\right)=8 c_{2} k-14 c_{2}{ }^{2}-k^{2}-(1-k)^{2} / 4 .
$$

The maximum $\left(c_{1}{ }^{*}, c_{2}{ }^{*}, c_{3}{ }^{*}\right)$ therefore occurs at $(3 k / 7,2 k / 7,2 k / 7)$ and

$$
g\left(c_{1}{ }^{*}, c_{2}{ }^{*}, c_{3}{ }^{*}\right)=k^{2} / 7-(1-k)^{2} / 4 .
$$

By inspection this maximum is negative outside of the range $1 \leqq k \leqq 4$. Therefore we need only look at values of $k$ in this range to seek a solution to (6). For these values it is easy to compute the maximum of the left side and the minimum of the right side of (6) independently. The details are omitted. Therefore (6) is never satisfied and $d_{G}$ is hypermetric.

To show that $d_{G}$ is nonHamming we exhibit an inequality that is satisfied by all Hamming extreme rays, but is not satisfied by $d_{G}$. We define 
$c \in R^{\left(\begin{array}{l}7 \\ 2\end{array}\right)}$ by

(7) $\quad c(i, j)=\left\{\begin{array}{rll}5 & i=1 & j=2,3 \\ -3 & i=1 & j \geqq 4 \\ 3 & i=2 & j=3 \\ -2 & i=2,3 & j \geqq 4 \\ 1 & i \geqq 4 & j \geqq 5\end{array}\right.$

We now check that

(8) $\sum_{i \perp j \in T} c(i, j) \leqq 0 \quad$ for all $T \in F_{n}$.

Recall that $1 \in T$ for every $T \in F_{n}$. The notation $[s, t]$ will refer to elements $T$ of $F_{n}$ that contain $s$ members of $\{2,3\}$ and $t$ members of $\{4,5,6,7\}$. The left side of $(8)$ is given in Table 2.1.

Table 2.1

\begin{tabular}{|c|c|c|c|c|c|c|}
\hline & 0 & 1 & 2 & 3 & 4 & $L H S$ of $(8)$ \\
\hline 0 & -2 & 0 & 0 & -2 & -6 & $-2+3 t-t^{2}$ \\
\hline 1 & -12 & -6 & -2 & 0 & 0 & $-12+7 t-t^{2}$ \\
\hline 2 & -28 & -18 & -10 & -4 & - & $-28+11 t-t^{2}$ \\
\hline
\end{tabular}

Therefore (3) is satisfied for all $T \in F_{n}$ hence $c x \leqq 0$ for all extreme rays $x$ of $H_{n}$ and hence for all $x \in H_{n}$. Since $c \cdot d_{G}=1, d_{G}$ is nonHamming.

It can be shown that the vector $c$ defined in (7) is in fact a facet of $H_{7}$. Inspection of Table 2.1 shows that equality holds in (3) for the subsets $T$ corresponding to $[s, t]=[0,1],[0,2],[1,3]$ and $[1,4]$. Now there are 4 subsets of the first type, namely, $\{1,4\},\{1,5\},\{1,6\}$ and $\{1,7\}$. Similarly there are 6 of the second type, 8 of the third type and 2 of the fourth for a total of $20=\left(\begin{array}{l}7 \\ 2\end{array}\right)-1$ subsets. The corresponding 20 extreme rays can be shown to be independent.

Thus the facets of the Hamming cone are not all of the type $b(i, j)=$ $c_{i} c_{j}(1 \leqq i<j \leqq n)$. This answers a question posed by Deza, who has given a list of facets of this type for $n \leqq 8[6]$.

3. Concluding remarks. The results of this paper first appeared in the author's Ph.D. thesis that is reprinted in part as [3]. Independently, P. Assouad [1] has shown that the Hamming cone is properly contained in the hypermetric cone using a different proof based on the corresponding dual cones. Many related topics may be found in the monograph in 
preparation by Assouad and Deza [2] that contains a survey of all known results on embeddability in $L^{1}$.

Acknowledgements. The author gratefully acknowledges Vaclav Chvátal for the supervision of this research which forms part of the author's Ph.D. thesis. The author also wishes to thank P. Assouad, M. Deza and I. Rosenberg for reading earlier versions of this paper and for providing many useful comments and preprints of their work. The suggestions of the referee are also acknowledged.

\section{REFERENCES}

1. P. Assouad, Un espace hypermétrique non plongeable dans un espace $L_{1}, \mathrm{C} . \mathrm{R}$. Acad. Sc. Paris 285 (1977), 361-363.

2. P. Assouad and M. Deza, Isometric embedding in $L_{1}$, in hypercubes and related problems, manuscript (1979).

3. D. Avis, On the Hamming cone, Technical report 77-5, Department of Operations Research, Stanford University, Stanford (1977).

4. - - On the extreme rays of the metric cone, Can. J. Math. 32 (1980), 126-144.

5. I. Blake and J. Gilchrist, Addresses for graphs, IEEE Trans. on Information Theory 19 (1973), 683-688.

6. M. Deza, Matrices de formes quadratiques non negatives pour des arguments binaries, C. R. Acad. Sc. Paris 277 (1973), 873-875.

7. M. Deza, On Hamming geometry of unitary cubes, (Russian), Doklady Akad. Nuak. SSR 134 (1960), 1037-1040.

8. _- Linear metric properties of binary codes, (Russian), Proc. 4th Soviet Union Conference on coding theory and transmission information, Moscow-Tashkent (1969), 77-85.

9. D. Ž. Djoković, Distance preserving subgraphs of hypercubes, J. Comb. Th. B 14 (1973), 263-267.

10. F. Harary, Graph theory (Addison-Wesley, Reading, Mass., 1969).

11. J. B. Kelly, Metric inequalities and symmetric differences, in Inequalities II (Academic Press, New York, 1970), 193-212.

12. - Hypermetric spaces, in Lecture Notes in Math. 490 (Springer-Verlag, 1975), 17-31.

13. J. Stoer and C. Witzgall, Convexity and optimization in finite dimensions I (SpringerVerlag, Berlin, 1970).

McGill University, Montreal, Quebec 